\title{
Rotigotine patches (Neupro) in early Parkinson's disease
}

Edited by AdRes Health Economics \& Outcomes Research

SYNTHETIC

DRUG

PROFILE

\section{INTRODUCTION}

Parkinson's disease (PD) is a neurodegenerative disorder secondary to the progressive loss of dopaminergic neurons in the substantia nigra (a portion of the midbrain responsible for movement initiation and coordination) and appearance of bradykinesia, resting tremor, rigidity and postural reflex impairment. The most common symptomatic therapy is levodopa, a dopamine precursor; however, long-term treatment leads to involuntary movements and response fluctuations which add to the complexities of later disease-management. Monotherapy with dopamine agonists may represent an alternative approach with a reduced likelihood of motor complications; these drugs, initially introduced as adjunctive therapy to levodopa, are less effective in controlling motor disability and tend to cause more side effects than levodopa itself.

\section{INDICATIONS AND DOSING}

Rotigotine is a new chemical substance belonging to the group of non-ergolinic dopamine agonists. This drug is used for the treatment of early-stage Parkinson disease as monotherapy, or with levodopa at all stages of the disease. The drug is available in transdermal patches of several sizes, containing different doses of rotigotine (Table I).

Recently, the Committee for Medicinal Products for Human Use (CHMP) of the EMEA issued a positive opinion on marketing authorization for rotigotine for idiopathic Restless Legs Syndrome (RLS) treatment. Rotigotine is currently included by AIFA (Agenzia Italiana del Farmaco) in a drug efficacy and safety monitoring program.

In early PD, the starting dose is $2 \mathrm{mg} / 24 \mathrm{~h}(4 \mathrm{mg} / 24 \mathrm{~h}$ for advanced PD), increased every week by $2 \mathrm{mg} / 24 \mathrm{~h}$ until an effective dose is reached, or up to a maximum dose of $8 \mathrm{mg} / 24 \mathrm{~h}(16 \mathrm{mg} / 24 \mathrm{~h}$ for advanced PD).

\begin{tabular}{ccccc}
\hline Size & $\mathbf{1 0 ~ \mathbf { ~ m } ^ { \mathbf { 2 } }}$ & $\mathbf{2 0 ~ \mathbf { ~ m } ^ { \mathbf { 2 } }}$ & $\mathbf{3 0 \mathbf { ~ m } ^ { \mathbf { 2 } }}$ & $\mathbf{4 0 ~ \mathbf { ~ m } ^ { \mathbf { 2 } }}$ \\
\hline Dose contained $(\mathrm{mg})$ & 4.5 & 9.0 & 13.5 & 18.0 \\
Dose released $(\mathrm{mg})$ & 2 & 4 & 6 & 8 \\
\hline
\end{tabular}

\section{Table I}

All available presentations for rotigotine transdermal application

\section{PHARMACOKINETICS}

The drug in the patch is constantly and dose-proportionately released over $24-\mathrm{h}$, resulting in constant plasmatic concentrations with daily application. The absorption of rotigotine is not influenced by gender, age, race, impaired renal or hepatic function. Rotigotine is widely distributed into tissues and extensively metabolized; the drug and its metabolites are mainly excreted in urine and less in bile and stool. 


\begin{tabular}{lll}
\hline Absorption & Bioavailability & High variability (1- 64\%), mean 37\% \\
& Cmax & $7 \mathrm{ng} / \mathrm{ml}$ after $1 \mathrm{mg} / \mathrm{Kg}$ administration \\
& Tmax & $15-18 \mathrm{~h}(1-27 \mathrm{~h})$ \\
& Binding to plasma proteins & $90.5-92.7 \%$ \\
& & \\
Metabolism and & Volume of distribution & $84 \mathrm{l} / \mathrm{kg}$ \\
distribution & Metabolism & Dealkyilation/ monoidroxilation, conjugation with glucuronic or sulphate acid \\
& Enzimes & CYP450: CYP2C19 \\
& Biological activity of metabolites & Several sulphotransferases and UDP-glucorosyltransferases \\
& Not present \\
& Clearance & $630 \mathrm{l} / \mathrm{h}$ \\
& Plasma terminal half-life & $5-7 \mathrm{~h}$ \\
& Elimination & $71 \%$ urine, 23\% bile and faeces \\
& Interactions & No interactions \\
\hline
\end{tabular}

Table II

Absorption, distribution, metabolism and elimination of rotigotine after transdermal administration

\section{FARMACODYNAMICS}

Rotigotine acts like a typical dopamine-agonist: this drug shows a close structural and functional analogy to dopamine, with a similar receptor binding and functional profile. Rotigotine is an agonist at all dopamine receptors, showing highest affinity and activity via the D3 receptor (the most widely cited receptor in the inhibition of locomotor activity).

\section{EFFICACY AND SAFETY}

The clinical development program for the rotigotine patch comprised four main studies (Phase III trials ): SP512 and SP513 to evaluate rotigotine efficacy in monotherapy in early-stage idiopathic PD; SP650 and SP515 to evaluate rotigotine efficacy in combination with levodopa in advanced idiopathic PD. SP512 and SP650 trials were placebo-controlled, whereas SP513 and SP515 were controlled against another dopamine agonist (ropinirole and pramipexole, respectively).

The primary efficacy end-point for monotherapy trials was based upon the UPDRS II/III score (Unified Parkinson's disease rating scale, a compilation of various PD assessment scales, the most frequently used for evaluating treatment responses), whereas for the evaluation of rotigotine in combination with levodopa the primary variable was the change in absolute time spent "off" (time during which PD symptoms are most prominent).

The safety profile of rotigotine can be considered typical of a dopamine-agonistic agent, with adverse reactions (AEs) like nausea, somnolence, vomiting, fatigue and constipation; the only exception is the high frequency of skin reactions in the application site. An increased frequency of AEs was observed in adjuvant therapy with levodopa, as compared to monotherapy.

\begin{tabular}{|c|c|c|c|c|}
\hline Study & Design & Comparator & Efficacy & Safety \\
\hline \multicolumn{5}{|c|}{ Monotherapy } \\
\hline \multirow[t]{2}{*}{ SP512 } & \multirow{2}{*}{$\begin{array}{l}277 \text { pts with early PD } \\
\text { randomized } 1: 1 \\
\text { Double-blind, multi center, } \\
\text { placebo-controlled }\end{array}$} & \multirow[t]{2}{*}{ Placebo } & $\begin{array}{l}\mathbf{2 0} \% \text { Improvement } \\
\text { P: } 19 \% \text { pts } \\
\text { Rt: } 48 \% \text { pts }(p<0.0001)\end{array}$ & \multirow{4}{*}{$\begin{array}{l}\text { AEs } \geq 5 \% \text { subjects: } \\
\text { P: } 57 \% \mathrm{pz} \\
\text { Rt: } 76 \% \mathrm{pz} \\
\text { Rp: } 70 \% \mathrm{pz} \\
\text { AEs typical of a dopamine agonist, } \\
\text { with an higher frequency of skin } \\
\text { reaction in the application site } \\
(P=14 \%, \mathrm{Rt}=37 \%, \mathrm{Rp}=8 \%)\end{array}$} \\
\hline & & & $\begin{array}{l}\text { Change in UPDRS II/III* score } \\
\text { P: } 1.31 \\
\text { Rt: }-3.98(p<0.0001)\end{array}$ & \\
\hline \multirow[t]{2}{*}{ SP513 } & \multirow{2}{*}{$\begin{array}{l}561 \text { pts with early PD } \\
\text { randomized 1:1:1 } \\
\text { Double-blind, multi center, } \\
\text { placebo-controlled, active } \\
\text { treatment }\end{array}$} & \multirow[t]{2}{*}{$\begin{array}{l}\text { Placebo, } \\
\text { ropinirole }\end{array}$} & $\begin{array}{l}\mathbf{2 0} \% \text { Improvement } \\
\text { P: } \mathbf{3 0 \%} \text { pts } \\
\text { Rt: } 52 \% \text { pts }(p<0.0001) \\
\text { Rp: } 70 \% \text { pts }(p<0.0001)\end{array}$ & \\
\hline & & & $\begin{array}{l}\text { Change in UPDRS II/III* score } \\
\text { P: }-2.33 \\
\text { Rt: }-6.83 \\
\text { Rp: }-10.78 \\
\text { Not showed non-inferiority to Rp }\end{array}$ & \\
\hline
\end{tabular}




\begin{tabular}{|c|c|c|c|c|}
\hline Study & Design & Comparator & Efficacy & Safety \\
\hline \multicolumn{5}{|c|}{ Adjoint to levodopa } \\
\hline \multirow[t]{2}{*}{ SP650 } & \multirow{2}{*}{$\begin{array}{l}351 \text { pts with advanced PD } \\
\text { ( } \geq 2.5 \text { h "OFF"/day) non } \\
\text { adequately controlled by } \\
\text { levodopa, randomized 1:1:1 } \\
\text { Double-blind, placebo- } \\
\text { controlled }\end{array}$} & \multirow[t]{2}{*}{ Placebo } & $\begin{array}{l}\text { Reduction in "off" time } \geq \mathbf{3 0} \% \\
\text { P: } 34.5 \% \\
\text { Rt } 18 \mathrm{mg}: 56.6 \%(p<0.001) \\
\text { Rt } 27 \mathrm{mg}: 55.1 \%(p<0.001)\end{array}$ & $\begin{array}{l}\text { AEs } \geq 5 \% \text { subjects: } \\
\text { P: } 62.6 \% \mathrm{pz} \\
\text { Rt: } 73.0 \% \mathrm{pz} \\
\text { Rp: } 70.3 \% \mathrm{pz}\end{array}$ \\
\hline & & & $\begin{array}{l}\text { Change in "off" time (hrs) } \\
\text { P: } 0.9 \\
\text { Rt 18mg: } 2.7(p<0.001) \\
\text { Rt 27mg: } 2.1(p=0.003)\end{array}$ & $\begin{array}{l}\text { AEs typical of a dopamine agonist, } \\
\text { with an higher frequency of skin } \\
\text { reaction in the application site } \\
\text { (P: } 20.1 \% \text {, Rt: } 39.9 \% \text {, Pr: } 11.9 \% \text { ) }\end{array}$ \\
\hline \multirow[t]{2}{*}{ SP515 } & \multirow{2}{*}{$\begin{array}{l}506 \text { pts with advanced PD } \\
\text { ( } \geq 2.5 \text { h "OFF"/day) non } \\
\text { adequately controlled by } \\
\text { levodopa, randomized 2:2:1 } \\
\text { Double-blind, placebo- } \\
\text { controlled. }\end{array}$} & \multirow[t]{2}{*}{$\begin{array}{l}\text { Placebo, } \\
\text { pramipexole }\end{array}$} & $\begin{array}{l}\text { Reduction in "off" time } \geq \mathbf{3 0} \% \\
\text { Pr: } 35 \% \\
\text { Rt: } 59.7 \%(p<0.001) \\
\text { P: } 67 \%(p<0.001)\end{array}$ & \\
\hline & & & $\begin{array}{l}\text { Change in "off" time (hrs) } \\
\text { P: } 0.88 \\
\text { Rt: } 2.46(p<0.0001) \\
\text { Pr: } 2.81(p<0.0001) \\
\text { Showed non-inferiority to Pr }\end{array}$ & \\
\hline
\end{tabular}

\section{Table III}

Summary of main studies investigating efficacy and safety of rotigotine monotherapy for early PD (SP512, versus placebo; SP513, versus placebo and ropinirole) or for advanced PD, in combination with levodopa (SP650, versus placebo; SP515, versus placebo and pramipexole)

* UPDRS score (Unified Parkinson's disease rating scale) in part II (daily activities) and III (motor evaluations) of questionnaire is the primary efficacy end-point for monotherapy trials; maximum score is $160(52+108)$ points (worst scenario) and the absence of disease signs is associated with a 0 score

AEs = adverse events $; \mathrm{P}=$ placebo; $\mathrm{Pr}=$ pramipexole; $\mathrm{Rp}=$ ropinirole; $\mathrm{Rt}=$ rotigotine

\section{ECONOMIC EVALUATIONS}

We were not able to retrieve any published economic evaluation on the use of rotigotine in Parkinson disease.

In table IV we calculated monthly pharmaceutical costs for available therapies for PD treatment. For each formulation we valorized minimum and maximum dosing (from the Summary Product Characteristics - SPCs) and DDD (Defined Daily Dose) for one month of therapy, using retail price. For packages with identical price we considered the less costly one. This is not to be intended as a cost-minimization analysis, but as a simple overlook of currently available treatments.

\begin{tabular}{|c|c|c|c|c|c|c|}
\hline \multicolumn{3}{|c|}{ Drug and dosing range } & \multirow{2}{*}{$\begin{array}{c}\text { Reimbursed } \\
\text { price } \\
\text { / package }\end{array}$} & \multicolumn{3}{|c|}{ Price of therapy (1 month) } \\
\hline \multirow{2}{*}{$\begin{array}{c}\text { Category } \\
\text { Anticholinergics }\end{array}$} & \multicolumn{2}{|l|}{ Active principle (DDD) } & & \multirow{2}{*}{$\frac{<}{3.2175}$} & \multirow{2}{*}{$\frac{>}{21.45}$} & \multirow{2}{*}{$\frac{\text { DDD }}{10.725}$} \\
\hline & biperidene (10 mg ) & $2 \mathrm{mg}$ & 4.29 & & & \\
\hline & & $4 \mathrm{mg}$ & 5.33 & 3.198 & 9.594 & 7.995 \\
\hline & & $5 \mathrm{mg}$ & 2.94 & 35.28 & 70.56 & 35.28 \\
\hline Antihistaminics & orfenadrine (200 mg) & $50 \mathrm{mg}$ & 2.12 & 2.544 & 10.176 & 5.088 \\
\hline \multirow[t]{12}{*}{ Levodopa and derivates } & levodopa + benserazide (600 mg) & $100+25 \mathrm{mg}$ & 4.71 & - & - & 28.26 \\
\hline & & $200+50 \mathrm{mg}$ & 16.84 & - & - & 30.312 \\
\hline & s.r. & $100+25 \mathrm{mg}$ & 5.56 & - & - & 33.36 \\
\hline & levodopa + carbidopa (600 mg) & $100+25 \mathrm{mg}$ & 4.98 & 8.964 & 23.904 & 17.928 \\
\hline & & $250+25 \mathrm{mg}$ & 6.68 & 2.004 & 32.064 & 9.6192 \\
\hline & s.r. & $100+25 \mathrm{mg}$ & 8.12 & 9.744 & 43.848 & 29.232 \\
\hline & s.r. & $200+50 \mathrm{mg}$ & 9.93 & 19.86 & 79.44 & 29.79 \\
\hline & m.r. & $100+25 \mathrm{mg}$ & 9.21 & 11.052 & 44.208 & 33.156 \\
\hline & m.r. & $200+50 \mathrm{mg}$ & 11.01 & 22.02 & 33.03 & 33.03 \\
\hline & melevodopa + carbidopa (600 mg) & $100 \mathrm{mg}$ & 9.12 & 27.36 & 54.72 & 54.72 \\
\hline & & $125 \mathrm{mg}$ & 9.12 & 9.12 & 54.72 & 43.776 \\
\hline & & $250 \mathrm{mg}$ & 9.12 & 9.12 & 54.72 & 21.888 \\
\hline
\end{tabular}




\begin{tabular}{|c|c|c|c|c|c|c|}
\hline \multicolumn{3}{|c|}{ Drug and dosing range } & \multirow{2}{*}{$\begin{array}{l}\text { Reimbursed } \\
\text { price } \\
\text { / package }\end{array}$} & \multicolumn{3}{|c|}{ Price of therapy (1 month) } \\
\hline Category & \multicolumn{2}{|c|}{ Active principle (DDD) } & & $<$ & $>$ & DDD \\
\hline Admantane derivates & amantadine $(200 \mathrm{mg})^{*}$ & $100 \mathrm{mg}$ & 7.1 & 10.65 & 21.3 & 21.3 \\
\hline \multirow{13}{*}{$\begin{array}{l}\text { Dopamine agonists } \\
\text { non ergot derived }\end{array}$} & \multirow[t]{2}{*}{ apomorphine } & $30 \mathrm{mg}$ & 21.67 & 65.01 & 650.1 & - \\
\hline & & $50 \mathrm{mg}^{\star \star}$ & 39.402268 & 118.2 & 472.8 & - \\
\hline & \multirow[t]{4}{*}{ rotigotine (6 mg) } & $2 \mathrm{mg}$ & 96.04 & 102.9 & 102.9 & 308.7 \\
\hline & & $4 \mathrm{mg}$ & 100.36 & 107.5286 & 107.5286 & 161.2929 \\
\hline & & $6 \mathrm{mg}$ & 130.47 & 139.7893 & 139.7893 & 139.7893 \\
\hline & & $8 \mathrm{mg}$ & 160.56 & 172.0286 & 172.0286 & 129.0214 \\
\hline & \multirow[t]{5}{*}{ ropinirole (6 mg) } & $0.25 \mathrm{mg}$ & 4.75 & 40.71429 & 244.2857 & 162.8571 \\
\hline & & $0.5 \mathrm{mg}$ & 8.68 & 37.2 & 223.2 & 148.8 \\
\hline & & $1 \mathrm{mg}$ & 10.44 & 22.37143 & 134.2286 & 89.48571 \\
\hline & & $2 \mathrm{mg}$ & 20.82 & 29.74286 & 118.9714 & 89.22857 \\
\hline & & $5 \mathrm{mg}$ & 44.04 & 31.45714 & 125.8286 & 75.49714 \\
\hline & \multirow[t]{2}{*}{ pramipexole (2.5 mg) } & $0.25 \mathrm{mg}$ & 16.52 & 33.04 & 297.36 & 165.2 \\
\hline & & $1 \mathrm{mg}$ & 65.16 & 65.16 & 293.22 & 162.9 \\
\hline \multirow{8}{*}{$\begin{array}{l}\text { Dopamine agonists } \\
\text { ergot derived }\end{array}$} & \multirow[t]{2}{*}{ cabergoline (3 mg) } & $1 \mathrm{mg}$ & 19.16 & 28.74 & 172.44 & 86.22 \\
\hline & & $2 \mathrm{mg}$ & 38.02 & 28.515 & 171.09 & 85.545 \\
\hline & \multirow[t]{2}{*}{ bromocriptine (40 mg) } & $5 \mathrm{mg}$ & 11.53 & 11.53 & 46.12 & 92.24 \\
\hline & & $10 \mathrm{mg}$ & 14.66 & 10.995 & 43.98 & 87.96 \\
\hline & \multirow[t]{3}{*}{ pergolide (3 mg) } & $0.05 \mathrm{mg}$ & 8.63 & 8.63 & 172.6 & 517.8 \\
\hline & & $0.25 \mathrm{mg}$ & 16.48 & 12.36 & 247.2 & 148.32 \\
\hline & & $1 \mathrm{mg}$ & 18.2 & 27.3 & 136.5 & 81.9 \\
\hline & dihydroergocryptine $(60 \mathrm{mg})$ * & $20 \mathrm{mg}$ & 34.88 & 26.16 & 313.92 & 156.96 \\
\hline \multirow[t]{4}{*}{ MAO B inhibitors } & \multirow[t]{3}{*}{ selegiline $(5 \mathrm{mg})^{*}$} & $1.25 \mathrm{mg}$ & 40.56 & 40.56 & 40.56 & 162.24 \\
\hline & & $5 \mathrm{mg}$ & 17.04 & 10.224 & 20.448 & 10.224 \\
\hline & & $10 \mathrm{mg}$ & 18.36 & 11.016 & 22.032 & 11.016 \\
\hline & rasagiline (1 mg) & $1 \mathrm{mg}$ & 135.55 & 145.2321 & 145.2321 & 145.2321 \\
\hline \multirow{2}{*}{$\begin{array}{l}\text { Other dopaminergic } \\
\text { substances }\end{array}$} & tolcapone (450 mg) & $100 \mathrm{mg}$ & 165.33 & 148.797 & 297.594 & 223.1955 \\
\hline & entacapone (1 g) & $200 \mathrm{mg}$ & 116.02 & 34.806 & 348.06 & 174.03 \\
\hline
\end{tabular}

\section{Table IV}

Monthly pharmaceutical costs for different available therapies for PD treatment (Informatore Farmaceutico 2008)

* Not in the Italian reimbursement list; ${ }^{* *}$ Ex-factory price

\section{PRODUCT OVERVIEW}

Name of the Medicinal Product Neupro

Marketing Authorisation Holder SCHWARZ PHARMA Ltd

Active Substance Rotigotine

Pharmaco-therapeutic Group Dopamine agonist

ATC Code N04BC09

Date of issue of Marketing Authorisation valid throughout the European Union 15 February 2006 


\section{REFERENCES}

- AIFA - Agenzia Italiana del Farmaco - Elenco dei farmaci sottoposti a monitoraggio intensivo available on http://www.agenziafarmaco.it/REGISTRAZIONE_FARMACO/sectionb998.html?target=\&area tematica=REGISTRAZIONE_FARMACO§ion_code=AIFA_FARMACOV_MONIT_INT\&cache_session=false

- DeRuiter J, Holston PL, DeRuiter AP. New Drug Review. PharmD US Pharm 2007; 32: 26-38

- Giladi N, Boroojerdi B, Korczyn AD, Burn DJ, Clarke CE, Schapira AH; SP513 investigators. Rotigotine transdermal patch in early Parkinson's disease: a randomized, double-blind, controlled study versus placebo and ropinirole. Mov Disord 2007; 22: 2398-404

- Hughes G, Burn D. Diagnosis and management of Parkinson's disease. Prescribe 2006

- Informatore Farmaceutico 2008, Ed. Elsevier Masson, updated to May, 2008

- Jankovic J, Watts RL, Martin W, Boroojerdi B. SP 512 Study Group. Transdermal rotigotine: double-blind, placebo-controlled trial in Parkinson disease. Arch Neurol 2007; 64: 676-82

- LeWitt PA, Lyons KE, Pahwa R; SP 650 Study Group. Advanced Parkinson disease treated with rotigotine transdermal system: PREFER Study. Neurology 2007; 68: 1262-7

- NEUPRO - EPAR summary for the public

- Neupro Scientific Discussion - European Medicines Agency (EMEA), 2006 available on http://www.emea. europa.eu/humandocs/PDFs/EPAR/neupro/062606en6.pdf

- Neupro Scientific Discussion - European Medicines Agency (EMEA), 2007 available on http://www.emea. europa.eu/humandocs/PDFs/EPAR/neupro/Neupro-H-626-II-03-AR.pdf

- Poewe WH, Rascol O, Quinn N, Tolosa E, Oertel WH, Martignoni E, et al.; SP 515 Investigators. Efficacy of pramipexole and transdermal rotigotine in advanced Parkinson's disease: a double-blind, double-dummy, randomised controlled trial. Lancet Neurol 2007; 6: 513-20

- Post-authorisation summary of positive opinion for Neupro - Committee for Medicinal Products for Human Use (CHMP) - European Medicines Agency (EMEA), 2008 available on http://www.emea.europa.eu/pdfs/human/ opinion/Neupro_17478808en.pdf 